Proceedings of SALT 28: 345-365, 2018

\title{
Some three students: towards a unified account of some*
}

\author{
Stephanie Solt \\ Leibniz-ZAS \\ Jon Stevens \\ The Ohio State University
}

\begin{abstract}
This paper investigates the semantics of the some+numeral construction (e.g. Some 20 cars were involved in the accident). Contra previous analyses, we demonstrate that some+numeral is not inherently approximative, but instead can be aligned to the canonical use of some as an indefinite determiner. Drawing on established theories of the semantics of degree expressions and of epistemic indefinites, we propose that on all of its uses, some encodes a domain-shifting function, which operates on sets of pluralities of some sort. We demonstrate that this analysis accounts for both the variable presence of an approximating effect as well as constraints on the distribution of some+numeral, and discuss consequences for the semantics of number and degree, and for the treatment of some more generally.
\end{abstract}

Keywords: numeral, degree, approximation, indefinite, domain shifting, kind

\section{Introduction}

The example in (1) illustrates a curious use of English some, on which it combines with a numerical expression.

(1) Some 20 cars were involved in the accident.

The goal of the present paper is to provide a semantic analysis of the 'some+numeral' construction, and to explore the consequences for the semantics of some more generally, and of numerical and degree expressions.

For many speakers, some+numeral has an approximating effect; that is, some 20 cars means something like about 20 cars. This has prompted semantic analyses that treat this some on par with approximators such as approximately and about. Sauerland \& Stateva (2007) analyze some $+n$ as denoting $n$ interpreted at the coarsest contextually available level of granularity, per (2), an analysis that renders some

* We would like to thank the audiences at SALT28, PLC41, XPrag2018 and the 91st LSA Annual Meeting, and at the ZAS and The Ohio State University, where versions of this work were presented. Particular thanks are due to Brandon Waldon for help with the online experiment. We acknowledge financial support from the German Science Foundation (DFG) to the first author (grant SO1157/1-2) and from the American Council of Learned Societies (ACLS) to the second author. 
equivalent to about. Such an approach does not however address what this some has to do with the ordinary indefinite determiner some. Anderson (2014) takes a more unified approach, proposing that some on its numerical use encodes a choice function $f$ operating on the set formed as the union of the precise denotation of the numeral and its pragmatic halo (Lasersohn 1999), per (3); this set is derived via a processes of coercion prompted by the need to satisfy an anti-singleton constraint characterizing some more generally. While the mechanism is different, Anderson's analysis, like Sauerland \& Stateva's, treats some+numeral as inherently approximative.

$$
\begin{aligned}
& \llbracket \text { some twenty } \rrbracket^{\text {gran }}=\llbracket \text { about twenty } \rrbracket^{\text {gran }}=\operatorname{coarsest}(\text { gran })(\llbracket \text { twenty } \rrbracket) \\
& \llbracket \text { some twenty } \rrbracket^{C}=f\left(\llbracket \text { twenty } \rrbracket \cup \text { halo }_{C}(\llbracket \text { twenty } \rrbracket)\right)
\end{aligned}
$$

In the present work, we argue that some+numeral is not first and foremost an approximating construction, though it has an approximate interpretation for many speakers. Like Anderson (2014), we align this occurrence of some to its canonical indefinite use; but departing from his analysis (and that of Sauerland \& Stateva), we demonstrate that it does not operate on degrees simpliciter but rather on individuals. More specifically, we argue that across all of its uses, some encodes a domain-shifting function that maps sets of individuals (of some sort) to sets of individuals.

The structure of the paper is as follows: In Section 2, we outline several properties of some+numeral that are not captured by an analysis along the lines of (2-3). Section 3 develops our proposal, and demonstrates how it accounts for the data in question. Section 4 investigates the relationship between domain manipulation, ignorance and approximation, and compares our account to that of Anderson (2014). Finally, Section 5 briefly outlines some broader consequences for the semantics of degree, and for the semantic analysis of indefinite some, and Section 6 concludes.

\section{Properties of some+numeral}

In this section, we review some properties of some+numeral that prove crucial to its correct analysis.

\subsection{Distributional restrictions}

In the original example (1), some composes with a measure of cardinality. Some may also occur with quantity measures in the mass domain (4), temporal extents (5), spatial extents (6), and certain adjectival measure phrases (7):

(4) Anna bought some 5 ounces of gold.

(5) a. Anna sang for some 45 minutes.

b. Anna moved to Berlin some 20 years ago. 
Some three students

(6) The tree is some 10 meters from the house.

(7) The path is some 20 feet long / longer than the driveway.

However, not all numerical expressions are possible. Prime examples of those that are not are expressions of temporal location such as those in (8).

a. * Anna started singing at some 3 o'clock. (cf. 'at about / roughly / approximately 3 o'clock')

b. * Anna moved to Berlin in some 1990. (cf. 'in about / roughly / around 1990')

In this respect, the behavior of some diverges from that of approximators such as about and roughly, which are perfectly acceptable as modifiers of such expressions. This alone is enough to suggest that some is not simply an ordinary approximator.

We also observe more subtle contrasts in acceptability such as the following, where again the behavior of some diverges from that of modifiers such as about:

a. The climbers ascended about / some 12,000 feet.

b. Base camp is located at about / some 12,000 feet above sea level.

c. Base camp is located at about / ?some 12,000 feet.

(10) a. The temperature rose some 20 degrees.

b. ?The temperature rose to some 20 degrees.

In (9a) and (9b), 12,000 feet describes a spatial extent, i.e. the distance between two points (in (9a), between the starting point and endpoint of the climbers' ascent; in (9b), between sea level and base camp). Here, modification by some is felicitous. In (9c), by contrast, the same numerical expression serves as a sort of coordinate, pointing to or naming a region in space (somewhat similar to describing base camp as being 'at the foot of the Khumbu glacier'). On this usage, modification by some is degraded, though about is acceptable. A similar contrast is observed in (10), which illustrates a difference between expressions of temperature change and those of location on the temperature scale. To be sure, both (9c) and (10b) can be made acceptable; but doing so seems to involve coercing their interpretations to extent-based ones (i.e. '12,000 feet above sea level'; '20 degrees above zero').

Considering the above examples as a whole, we may observe that the numerical expressions that are felicitous with some have in common that they can be construed as referring (in a pre-theoretical sense) to plural individuals of some sort. Cardinal numerals have been analyzed as cardinality predicates (Landman 2004); twenty, on this view, describes a plural individual formed as the mereological sum of 20 atomic individuals. By the same token, five ounces can be interpreted as describing a portion of matter equal in weight to the concatenation of five 1-ounce portions. The 
examples in (5-7) can be understood as referencing more abstract sorts of individuals: temporal extents, spatial extents (vectors), and scale segments. For example, twenty meters can be thought of as a spatial extent which can be subdivided into 201 -meter extents, and 45 minutes as a temporal interval consisting of 45 non-overlapping 1-minute intervals. By contrast, 3 o'clock is not three of anything, nor is 1990 interpretable in any non-arbitrary sense as an individual made up of 1,990 atomic parts. Rather, temporal expressions such as these seem to directly refer to or name points or intervals on the timeline, just as 12,000 feet in (9c) refers to a point or region in space.

The notion that some is restricted to composing with measure expressions understood as individuals receives further support from the fact that it is likewise disallowed in contexts in which numerical expressions directly denote degrees. The clearest case of this involves mathematical statements. As illustrated in (11a), approximators are fine in such contexts. But some is quite bad; faced with (11b), one is tempted to ask 'some 100 of what?'. Example (12) makes a similar point

(11) a. Seven times fourteen is about / roughly / approximately one hundred. b. ?? Seven times fourteen is some one hundred.

(12) Five kilos equals roughly / ??some eleven pounds.

A similar pattern is observed in answers to questions that ask for a number:

(13) Q: What is the largest number of participants you've had in a seminar?

A: Around / ??some 90.

Taken as a whole, the distributional constraints outlined here provide evidence that unlike approximators such as about, some does not operate in the domain of degrees per se, but instead composes with numerical expressions when they denote individuals of some sort. This speaks against an analysis along the lines of (2) and (3), both of which start from a basic degree-based denotation of the numeral.

\subsection{Non-approximating uses}

The previous proposals for the semantics of some+numeral represented in (2) and (3) have started from the assumption that the construction has an inherently approximate interpretation. The issue with this is that although some+numeral has an approximating effect for many speakers, it may also be used to convey a precise value. This is illustrated by the naturally occurring examples below (from the Corpus of Contemporary American English; Davies 2008-), in which some composes with a non-round number:

(14) a. Of some 206 students who responded to the survey, $52 \%$ were female. 
Some three students

b. Windstar offers an atlas-size roster of ports of call - some 144 of them in nearly 50 nations throughout Europe and the Americas.

c. As a part of the PMA process, we filed biosafety data covering some 329 individual, discrete biosafety studies.

d. In 1986, 459,369 CPFV anglers landed some 2,835,021 fish.

In all of these cases, the most plausible intended meaning is the precise one. The writer of (14a), for example, was knowledgeable about and intended to convey the exact number of students who responded, namely 206. In each example, replacing some with about would change the meaning substantially.

In order to test the hypothesis that some+numeral is not necessarily interpreted approximately, we carried out a small-scale online experiment. The goal was to determine the extent to which speakers assign approximate versus precise interpretations to some+numeral in comparison to about+numeral and bare numerical expressions, and also to assess how this interacts with the roundness of the modified numeral, as this factor is known to play a role in the availability of approximate readings more generally (Krifka 2002, 2007).

A total of 72 native English speakers were recruited via Amazon Mechanical Turk, and were shown sentences of the form in (15), with the task being to indicate the interpretation of the numerical value by filling in the lower and upper bounds:

The storm caused about/some/ $\varnothing 100 / 103$ homes to lose power.

How many homes lost power because of the storm?

Between and

Three modifier conditions were tested (some / about / bare) in two numerical conditions (round / non-round). Participants' responses were coded as either EXACT (upper and lower values differ by $\leq 1$ from the value in the stimulus sentence) or APPROXIMATE (upper and/or lower values differ by $>1$ from stimulus value).

The results are shown in Figure 1. As seen here, responses to the about and bare conditions were as might be expected: about $n$ was interpreted almost exclusively as approximate, while bare $n$ was interpreted in the great majority of cases as exact. Some patterned distinctly from both (some vs. bare: $\mathrm{z}=7.8, \mathrm{p}<0.001$; some vs. about: $\mathrm{z}=-4.2, \mathrm{p}<0.001$ ), being interpreted primarily as approximate with round numbers, but eliciting a mixture of approximate and exact responses in combination with non-round numbers. At the respondent level, a variety of patterns were observed. The most common (33 out of 72 respondents) was to interpret both about and some approximately across the board, but bare exactly in one or both roundness conditions. But the second most common pattern (16/72 respondents) was to give approximate responses for about and some+round and exact responses to bare and 


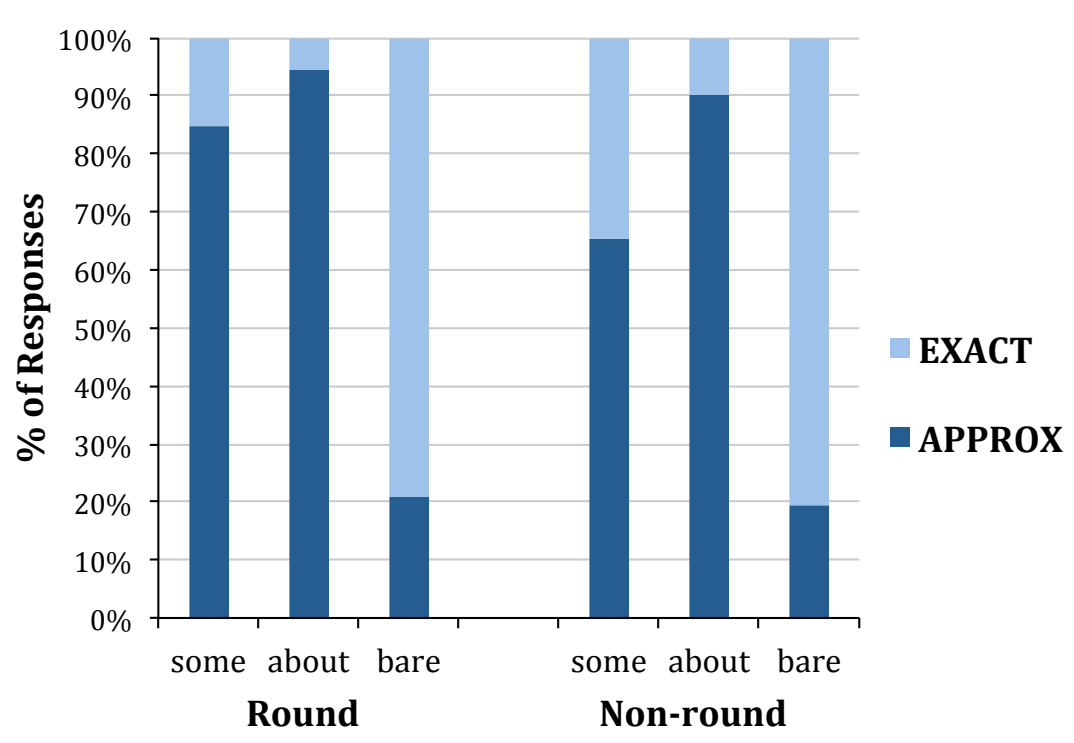

Figure 1 Results of online experiment.

some+non-round; an additional minority (6/72) treated about as approximate and both some and bare as exact across roundness conditions. The remaining respondents did not distinguish the three modifier conditions, or gave other sorts of responses. In total, nearly a third of respondents interpreted some+numeral as exact in at least one condition where about+numeral was interpreted approximately.

The corpus data and experimental results thus provide evidence that some+numeral is not always used and interpreted approximately, at least for some speakers. ${ }^{1}$ A precise interpretation is possible in particular with non-round numbers. This is not captured by an analysis along the lines of (2) or (3), on which the core semantic function of some is to force an approximate interpretation of the numeral it composes with. On the other hand, there is clearly speaker variation in this area, and this too requires explanation.

An obvious question that arises is what the semantic contribution of some actually is, in those cases where it does not add approximation. What is the difference between, say, The accident resulted in some 27 deaths and its bare counterpart The accident resulted in 27 deaths, or between The tree is some 11 meters from the house and the simpler The tree is 11 meters from the house? As far as we are able to diagnose, there is no truth-conditional difference, though there are some pragmatic

1 Based on anecdotal evidence, we have the impression that it is older English speakers who tend to allow the non-approximate use, whereas younger speakers tend to assign an approximate interpretation across the board. We have not however tested this experimentally. 
Some three students

effects. In particular, the some versions have an emphatic effect, highlighting the magnitude of the numerical value, or perhaps the precision at which it is reported. In Stevens \& Solt 2018 we provide a pragmatic account of these effects, based on Horn's division of pragmatic labor (Horn 1984). But in terms of the semantics, the clearest effect of adding some is to restrict the contexts in which a numerical expression may occur. It is this effect, as well as the varying presence of approximation, that we seek to account for in the present paper.

\section{Proposal}

In this section we develop a semantic analysis of the some+numeral construction. We begin with our assumptions regarding the semantics of the component parts, namely numerals and some itself, and then demonstrate how this allows a compositional analysis of some+numeral that accounts for the distributional and interpretive facts outlined in the previous section.

\subsection{Building blocks}

Number words Number words have received a wide variety of semantic analyses, being treated alternately as generalized quantifiers (Barwise \& Cooper 1981), predicates (Landman 2004), modifiers (Ionin \& Matushansky 2006) and degreedenoting expressions (Nouwen 2010). Several authors have proposed that they have denotations at two or more distinct semantic types related by type shifting rules, interpretable for example as both quantifiers and predicates (Geurts 2006), or as degrees and quantifiers over degrees (Kennedy 2015). We adopt one such dual-type approach, namely that of Rothstein $(2012,2013,2017)$, according to which number words have denotations as both predicates and arguments (singular terms). On the predicative interpretation, they are cardinality predicates (type $\langle e, t\rangle$ ), that is, equivalence classes of pluralities composed of a specified number of atoms, per (16a). The argument interpretation is formed via nominalization of the corresponding predicate, per (16b), and may be thought of as type $d$, the type of degrees. ${ }^{2}$

$$
\begin{aligned}
& \text { a. } \llbracket \text { three }_{\langle\text {et }\rangle} \rrbracket=\{x:|x|=3\} \\
& \text { b. } \llbracket \text { three }_{d} \rrbracket=\cap\{x:|x|=3\}=3_{d}
\end{aligned}
$$

The duality exhibited in (16) is parallel to that widely assumed to obtain in the nominal domain (Chierchia 1998), according to which bare plurals and mass nouns have denotations as both kinds (singular terms) and properties. This connection

2 Rothstein in fact uses $n$ as the type of numbers as singular terms; we however assume that numbers in this sense are a sort of degree. 
between degree and kind expressions is explored in depth by Anderson \& Morzycki (2015) and more recently Scontras (2017), whose proposal we will return to in Section 5 below.

Some An important insight that comes out of the literature on epistemic indefinites is that the semantic import of indefinite determiners of various sorts can be understood as manipulating or constraining domains of quantification (Kadmon \& Landman 1993; Kratzer \& Shimoyama 2002; Alonso-Ovalle \& Menéndez-Benito 2003, 2010, 2011, 2013). Kratzer goes as far as to suggest that "quite generally, indefinite determiners might be domain shifters, operations on quantification domains" (Kratzer 2005: 134). Some such items, such as English any and German irgendein, widen quantificational domains; others, such as Spanish algún, potentially narrow domains to a contextually relevant subset (sometimes with additional constraints). Domain-related operations have been invoked to account for ignorance and free choice effects, specific interpretations (Schwarzschild 2002), and even polarity sensitivity (Kadmon \& Landman 1993; Chierchia 2013).

Drawing on this literature, and in particular on the body of work by AlonsoOvalle \& Menéndez-Benito, we propose that some on all of its uses encodes a variable $f$ over functions from sets (domains) to sets. We further assume a nonquantificational approach in which this function is the sole semantic content of some; quantificational force arises via existential closure (Heim 1982 and ff.). We thus propose the following (preliminary) lexical entry:

\section{Lexical entry for some (preliminary):}

$$
\llbracket \text { some }_{\langle\alpha t, \alpha t\rangle} \rrbracket=\lambda P_{\langle\alpha t\rangle} \lambda x_{\alpha} . f(P)(x)
$$

On its indefinite determiner use, some takes the nominal expression as argument, per (18). The resulting predicative expression composes by set intersection with the sentential predicate; after existential closure, we obtain (19) as the semantics of a simple 'quantificational' example.

$$
\begin{aligned}
& \llbracket \text { some students } \rrbracket=\llbracket \text { some } \rrbracket(\llbracket \text { students } \rrbracket) \\
& =\lambda x . f(\lambda y . \text { students }(y))(x)
\end{aligned}
$$

$$
\begin{array}{ll}
\text { Some students called. } & \\
\exists x[f(\lambda y . \text { students }(y))(x) \wedge \text { called }(x)] & f(P) \subseteq P
\end{array}
$$

We assume that in the default case, the function $f$ is interpreted as a subset function, i.e., a function that maps a set to one of its subsets (not necessarily a proper one). Thus (19) states that some members of a contextually determined set of students called. Yet in the case where the hearer does not know which set of student pluralities the speaker has in mind, the communicative effect of (19) is not distinguishable from 
Some three students

what would obtain via quantification over the entire set of pluralities of students.

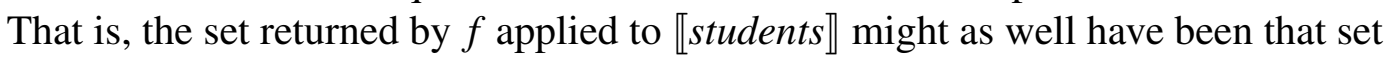
itself. This accounts for the intuition that there is no obvious difference in truth conditions between (19) and the simpler Students called.

\subsection{The semantics of some+numeral}

With the above pieces in place, we are now in a position to provide a compositional analysis of some+numeral. We begin with the simpler case of an unmodified numeral. On the prenominal use, we propose that the numeral takes on its predictive interpretation (type $\langle e, t\rangle$ ), allowing it to compose with the nominal predicate via set intersection, per (20). ${ }^{3}$ Following further composition with the sentential predicate and existential closure (as in the above case of a plural indefinite) we derive (21) for a simple numerical example.

$$
\begin{aligned}
& \llbracket \text { twenty cars } \rrbracket=\llbracket \text { twenty } \\
& \langle\text { et }\rangle \rrbracket \cap \llbracket \text { cars } \rrbracket \\
& =\lambda x . \operatorname{cars}(x) \wedge|x|=20
\end{aligned}
$$

(21) Twenty cars were involved in the accident.

$$
\exists x[\operatorname{cars}(x) \wedge|x|=20 \wedge \text { in-accident }(x)]
$$

In the case of some+numeral, we propose that some as defined in (17) first composes with the numeral, which again has its predicative interpretation. Composition then proceeds exactly as in the unmodified numeral case, yielding (24) as the interpretation of a relevant example.

$$
\begin{aligned}
& \llbracket \text { some twenty } \rrbracket=\llbracket \text { some } \rrbracket\left(\llbracket \text { twenty }_{\langle\text {et }\rangle} \rrbracket\right) \\
& =\llbracket \text { some } \rrbracket(\{y:|y|=20\}) \\
& =\lambda x . x \in f(\{y:|y|=20\}) \\
& \llbracket \text { some twenty cars } \rrbracket=\llbracket \text { some twenty } \rrbracket \cap \llbracket \text { cars } \rrbracket \\
& =\lambda x \cdot \operatorname{cars}(x) \wedge x \in f(\{y:|y|=20\})
\end{aligned}
$$

(24) Some twenty cars were involved in the accident.

$$
\exists x[\operatorname{cars}(x) \wedge x \in f(\{y:|y|=20\}) \wedge \text { in-accident }(x)]
$$

On the analysis developed here, the semantics of some+numeral is thus stated in terms of a function $f$ that maps the predicative or set-based denotation of a numeral - here, twenty - to some other set. Crucially, this formal device gives us a path towards accounting for the distributional and interpretive facts that were discussed in the previous section. We turn to this in the remainder of this section.

3 We leave open the possibility that there might also be other routes by which an unmodified prenominal numeral can compose with a substance noun. For example, the numeral might be interpreted at its argument type (type $d$ ), with a null measurement element playing the role of linking degrees to individuals. Nothing in the present analysis hinges on whether or not such a second route is available. 


\subsection{Explaining the data}

Variable approximating effect As discussed in Section 2, many speakers interpret some+numeral approximately, but others assign it a precise interpretation, at least in some contexts. We propose that this variation may be explained with reference to different values that the function $f$ may take, as depicted below:

a. Basic some
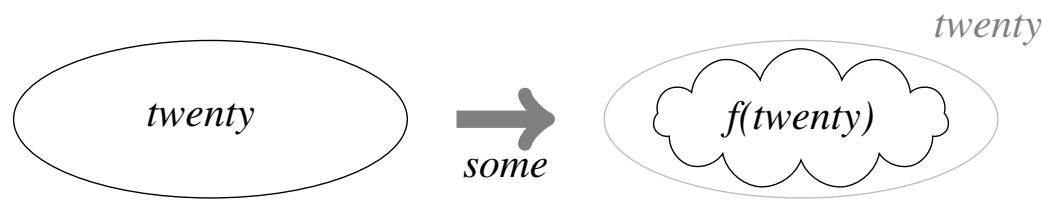

\section{b. Approximating some}

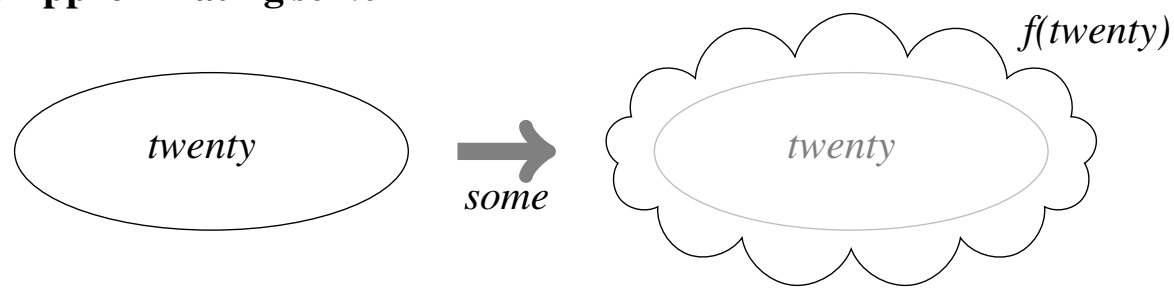

The non-approximating reading arises when the function $f$ is interpreted as a subset function, as was the case for indefinite determiner some: The set of individuals of a given cardinality (e.g. 20) is mapped to one of its (not necessarily proper) subsets. Similarly to what was observed in the indefinite determiner case, on this interpretation the some+numeral example in (24) is truth-conditionally indistinguishable from the corresponding bare numeral example in (21), though as noted earlier there are certain pragmatic effects. The approximating reading by contrast arises when $f$ maps a cardinality-based set to a superset containing also pluralities close in cardinality to that of the specified value. For example, the set of plural individuals made up of 20 atoms (i.e., the set that is the predicative denotation of twenty) might be mapped to the set containing pluralities of cardinality 18,19,20,21 or 22. With $f$ valued in this way, the example in (24) has an approximate interpretation, roughly equivalent to what would obtain if some were replaced by about.

Our online experiment provided evidence that speakers vary with respect to which of the two interpretations they have: Some (probably the majority) have the approximating interpretation arising as in (25b), while others have the nonapproximating interpretation in (25a). Furthermore, even among speakers who have the approximating some, there seem to be two possibilities. Recall the experimental finding that some speakers appear to allow an approximate reading for some+numeral only in combination with a round number, whereas a non-round numeral modified 
Some three students

by some is assigned an an exact interpretation. This actually parallels an interpretive possibility available to bare numerals: As pointed out in particular by Krifka (2002, 2007), round numbers themselves may be interpreted imprecisely, while non-round numbers are necessarily interpreted precisely. Suppose we take a semantic view of numerical imprecision, according to which a sentence such as Twenty cars were involved in the accident has a reading on which it is actually true if the number of cars involved was close to but not exactly equal to 20 (Krifka 2007; Sauerland $\&$ Stateva 2007; Solt 2014). Then the 'round only approximate' interpretation of some+numeral, for those speakers who have it, amounts to interpreting the numeral in the broadest possible manner compatible with its semantics (approximate for round numbers, precise for non-round numbers). This is strikingly parallel to the domainwidening effect which has been proposed to characterize indefinite determiners such as any and German irgendein (Kadmon \& Landman 1993; Kratzer \& Shimoyama 2002). We take this as a point in favor of the present approach of aligning some to indefinite determiners more broadly. For other speakers, though, some has an approximating effect across the board. For these speakers we propose that some has the effect of widening the interpretation of the numeral independently of what sort of interpretation its bare counterpart would allow.

Here we must note that the domain widening that we propose is responsible for the approximating effect is restricted to the numerical use of some, and is not available on the ordinary indefinite use. Some cars, for example, can never be interpreted as a set that contains not just cars but also entities that are in some respect similar to cars. Thus the some of some+numeral is at least for many speakers not identical to indefinite some, but rather involves a further elaboration of the basic template in (17). Yet this development is made possible by the basic meaning of some, whose core content is the underspecified function $f$ that manipulates sets; the step to the widening (i.e. approximating) function is a small one. As to why it is the numerical use on which $f$ can map sets to supersets, we hypothesize that this has to do with the nature of the sets involved. On its predicative interpretation, twenty tells us nothing about the nature of the individuals in question, but only about their cardinality. We suspect that for this reason, it is implausible for this set to be restricted contextually; but a principled basis for widening is make possible by the structure of the number line, which determines which entities are similar to those in the original set.

Distributional restrictions A second finding from Section 2 was that some is restricted with respect to the numerical expressions it may compose with, and the contexts in which the resulting some+numeral construction may occur. To repeat the crucial data, we observed that some is acceptable with measure expressions such as those in (26), but is ungrammatical with those such as (27): 
(26) a. Anna bought some 5 ounces of gold.

b. Anna sang for some 45 minutes.

c. The tree is some 10 meters from the house.

d. The path is some 20 feet long / longer than the driveway.

a. *Anna started singing at some 3 o'clock.

b. *Anna moved to Berlin in some 1990.

This pattern falls out directly from the semantics we have proposed for some. On our analysis, some takes as its first argument a set of individuals of some sort. This has the consequence that it is restricted to composing with set-denoting expressions. This requirement is obviously satisfied in the indefinite determiner use: The substance noun denotes a set of individuals (singular or plural), and the result of composition with some is a possibly restricted domain over which existential quantification operates (see (19)). On the approach we have adopted to the semantics of numerals, these too have (in one of their instantiations) the proper semantic type to be selected by some. It is this that allowed a compositional analysis of the cardinality variety of the some+numeral construction, per (22-24).

The same approach can be extended to the examples in (26). Consider first the mass expression in (26a). Just as twenty can be construed as the set of pluralities composed of 20 atomic individuals, five ounces can be construed as the set of portions of matter weighing 5 ounces. Compositionally, this can be achieved by analyzing ounce in terms of an OUNCE measure function, which maps individuals to their weight in ounces (Rothstein 2011, 2012, 2017). The first argument slot of ounce can be saturated by a numeral on its type $d$ interpretation, yielding an expression of set type that can serve as argument to some. Finally, just as in the numerical case, the resulting predicate can compose via set intersection with a substance noun:

$$
\begin{aligned}
& \llbracket \text { ounce } \rrbracket=\lambda d \lambda x_{e} \cdot \mu_{\text {OUNCE }}(x)=d \\
& \llbracket 5 \text { ounces } \rrbracket=\lambda d \lambda x_{e} \cdot \mu_{O U N C E}(x)=d\left(5_{d}\right) \\
& =\lambda x_{e} \cdot \mu_{O U N C E}(x)=5 \\
& \llbracket \text { some } 5 \text { ounces } \rrbracket=\lambda y \cdot y \in f\left(\lambda x_{e} \cdot \mu_{O U N C E}(x)=5\right) \\
& \llbracket \text { some } 5 \text { ounces of gold } \rrbracket=\lambda y \cdot g o l d(y) \wedge y \in f\left(\lambda x_{e} \cdot \mu_{O U N C E}(x)=5\right)
\end{aligned}
$$

Turning to the measure expressions in (26b-d), there are well-established semantic theories that treat these as denoting sets of individuals of a more abstract nature. In particular, in theories of temporal semantics such as that of Krifka (1989), expressions of duration can be analyzed as predicates or sets of temporal intervals $t$, which may describe the runtime of an event $e .45$ minutes, for example, is the set of intervals of length 45 minutes. Given the type-flexible semantics proposed for 
Some three students

some in (17), such an expression is of the correct semantic type to saturate its first argument slot. The effect is to map the original set of intervals to some subset or superset. An example such as (26b) can then be analyzed as in (35):

a. $\llbracket 45$ minutes $\rrbracket=\{t:$ minutes $(t)=45\}$

b. $\llbracket$ some 45 minutes $\rrbracket=\llbracket$ some $\rrbracket(\llbracket 45$ minutes $\rrbracket)$ $=\lambda t^{\prime} \cdot t^{\prime} \in f(\{t:$ minutes $(t)=45\})$

(33) Sue sang for some 45 minutes.

$\exists e[$ singing $(e) \wedge \operatorname{Agent}(e$, Sue $) \wedge \tau(e) \in f(\{t:$ minutes $(t)=45\})]$, where $\tau(e)$ is the runtime of the event $e$

Somewhat similarly, in Vector Space Semantics (Zwarts 1997; Zwarts \& Winter 2000), spatial extents are conceptualized as vectors, that is, directed line segments between points in space. Spatial measure phrases such as ten meters are analyzed as denoting sets of such vectors, which compose intersectively with the expressions they modify. Again, such sets can serve as arguments of some, yielding (35) as the logical form for a relevant example.

$$
\llbracket 10 \text { meters } \rrbracket=\{v:|v|=10 m\}
$$

The tree is some 10 meters from the house.

$$
\exists u[u \in f(\{v:|v|=10 m\}) \wedge \text { start }(u, \text { house }) \wedge \text { end }(u, \text { tree })]
$$

Authors including Faller (2000) and Winter (2001) demonstrate that the Vector Space Semantics framework can be extended also to adjectival measure phrases such as those in (26d), with in this case the vectors in question being essentially segments of a measurement scale. Thus in this case too, we have an expression of the correct semantic type to compose with some.

However, not all numerical expressions have denotations at the necessary type, an example being expressions of temporal location such as those in (27). While theories of temporal semantics are not united on this point, an established view is that such expressions are referential (see Altshuler 2014 and references therein). For example, 1990 can be analyzed as directly referring to an interval on the timeline, just as, say, Barack Obama refers to an ordinary individual. Something like three o'clock is underspecified as to the date and day part (am or pm) in question, but once this parameter is filled in, it too can be treated as referential.
a. $\llbracket 1990 \rrbracket=1990$
b. $\llbracket 3 o^{\prime}$ clock $\rrbracket=3: 00_{i}$

Thus we correctly predict that some will be ruled out with such expressions due to a type clash, just as it is with referential expressions in the nominal domain (e.g. *some Barack Obama, *some he). 
Independent evidence for the type distinction invoked here is provided by the contrasts in (37) vs. (38). Numerically modified plural noun phrases as well as expressions of the sort in (26) can serve as the restrictor for every. But temporal locative expressions such as those in (27) cannot, even if the intended meaning is an entirely plausible one. We might expect that three o'clock could denote the set of all time points that are labeled 3:00 (two per day), and 1990 the set of points within the year 1990. However, such sets are not available as the restrictor of every; to express the intended meanings, the examples must be modified as shown to include an appropriate set-denoting expression. We take this to show that the latter type of numerical expressions, unlike the former, do not have a set-based interpretation. It is this that explains the impossibility of modification by some.

a. There were three teachers for every ten students.

b. For every 5 ounces of gold you collect, you receive a prize.

c. A bell rings every 45 minutes.

d. There is a signpost every 10 meters.

a. A bell rings *every 3 o'clock / every afternoon at 3 o'clock.

b. There were humanitarian forces in the country *at every 1990 / at every point in 1990 .

Recall finally that on our account, cardinal numerals can be shifted from a predicative to an argumental (type $d$ ) interpretation via the 'down' operator $\cap$. We assume that this is also possible for measure expressions of the sort in (26), e.g. 5 ounces, 45 minutes and the like. In all of these cases, this allows such expressions to occur in positions that require degrees, such as in particular mathematical statements (see Section 2). We then correctly predict that such uses are incompatible with some, which can only compose with a numerical expression on its predicative interpretation (e.g. ??Five kilos equals some eleven pounds).

\section{Domains, ignorance and approximation}

In Section 3, we related the properties of the some+numeral construction to the presence of a domain-shifting function $f$ in the lexical entry of some. A proposal very much along the same lines was made previously by Anderson (2014). We compare the two accounts here.

As discussed earlier, Anderson proposes that some on its numerical use lexicalizes a choice function that operates on the set formed as the union of the precise denotation of the numeral and its pragmatic halo (see (3)). This approach is motivated by the idea that some more generally encodes an anti-singleton constraint on its domain of quantification, meaning that the domain cannot be a singleton set. The 
Some three students

notion of an anti-singleton constraint derives from the work of Alonso-Ovalle \& Menéndez-Benito (2003, 2010, 2011, 2013), who invoke it to account for ignorance effects with the Spanish indefinite determiner algún 'some'. By way of example, (39) would be infelicitous if the speaker knew which doctor María had married, as evidenced by the impossibility of the continuation in parentheses. To account for this effect, Alonso-Ovalle \& Menéndez-Benito propose that algún lexicalizes a subset selection function with the property that its output is always a non-singleton set, per (40). The ignorance inference is derived as an implicature relative to singletondomain alternatives that the speaker could have (but did not) utter, the effect of which is to convey that it is not the same doctor in all of the speaker's belief worlds.

(39) María se casó con algún médico (\# en concreto con el Dr. Smith). 'María married some doctor or other (\# namely Dr. Smith)'

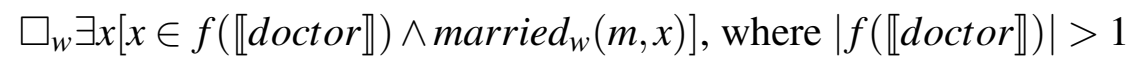

Weir (2012) extends this analysis to English some, and Anderson applies it to some+ numeral. Crucially, on Anderson's analysis some on this use composes with a set of numbers, i.e. degrees. If the numeral has its precise denotation this is necessarily a singleton set; it is only by coercing the denotation of the numeral to an imprecise one that the anti-singleton requirement is satisfied.

Our account differs from Anderson's in that the some of some+numeral operates not on sets of numbers but rather on sets of plural individuals, which may (and indeed likely do) have multiple members. The approximative effect does not arise as the result of an obligatory process of coercion, but rather relates in a more general way to the core semantic content of some, which is stated in terms of domain shifting. We believe that this gives our approach an advantage in two respects. First, it allows an explanation for the observation from Section 2 that some does not always have an approximating effect; on Anderson's account, such an effect is an obligatory consequence of the basic semantics of some. A second advantage involves the extension of the account beyond English. In particular, in Spanish, it is not the epistemic indefinite determiner algún but rather the ordinary indefinite un that has an approximating effect with numerical expressions (Luisa Martí, p.c.). This argues against aligning the approximating function directly to the epistemic effect observed with certain indefinite determiners, and instead treating it as one possible consequence of the basic domain-shifting function of indefinites.

This being said, our account is not incompatible with some encoding an antisingleton function. The relevant pattern is that below:

(41) a. Some student called, \#namely Anna.

b. Some students called, namely Anna, Berta and Carla.

c. Some three students called, namely Anna, Berta and Carla. 
In combination with a singular noun, some gives rise to an ignorance effect. With a plural noun, this effect is absent. Some+numeral patterns with the plural case. We may account for this pattern by positing the following revised entry for some, based on Alonso-Ovalle \& Menéndez-Benito's analysis of algún:

\section{Lexical entry for some (final):}

$$
\llbracket \text { some }_{\langle\alpha t, \alpha t\rangle} \rrbracket=\lambda P_{\langle\alpha t\rangle} \lambda x_{\alpha} . f(P)(x) \text {, where for all } P,|f(P)|>1
$$

The ignorance effect characterizing singular some in (41a) can then receive a parallel analysis to the Spanish example (39). Furthermore, the lack of an ignorance inference with some+numeral in (41c) can be explained via the very same logic that Alonso-Ovalle \& Menéndez-Benito apply to plural algunos, which like English some+plural noun does not give rise to an ignorance inference. Specifically, the anti-singleton constraint can be satisfied at the level at which some combines with the numeral three, but intersection with the substance noun students may nonetheless produce a singleton set (this would be the case for example if $f$ in (41c) returned the set containing the student plurality anna $\oplus$ berta $\oplus$ carla plus some triple of nonstudents). In this case, no implicatures are generated, because any singleton-domain alternative is equivalent to a non-singleton-domain utterance, which could have been the one intended by the speaker. The simple plural case in (41b) turns out to be more problematic: Alonso-Ovalle \& Menénendez-Benito's analysis of plural algunos rests crucially on there being semantically contentful plural morphology on the indefinite determiner, which is not the case in English. One potential explanation is that in a non-quantized set of pluralities, the anti-singleton constraint is in some way trivially satisfied; due to space considerations we cannot pursue this issue in depth.

We note also that while the semantics of some do not require that a full noun phrase like some three students denote a non-singleton set, this nonetheless appears to be the preferred interpretation. Some+numeral tends to have an anti-specificity effect: While (43a) is easily interpreted to mean that Anna and Zoe danced with the same three Belgian students (i.e. specifically), (43b) suggests that it was a different three such students in the two cases. Thus it appears that we infer from the presence of some that there is more than one contextually relevant triple of Belgian students.

At the party ...

a. Anna danced with three Belgian students, and Zoe did too.

b. Anna danced with some three Belgian students, and Zoe did too.

To summarize, while approximation and ignorance effects are on our account not directly connected (contra Anderson 2014), they nonetheless have the same underlying source, namely the domain shifting operation that is a primary semantic function of indefinite determiners. Approximation is thus one possible effect of domain manipulation. 
Some three students

\section{Broader consequences}

Our primary focus in this paper has been to provide a semantic analysis of the some+numeral construction. In this section, we briefly consider some broader consequences for the semantics of some, and of degree expressions.

Our analysis rests on a particular approach to the semantics of numerals and measure expressions, according to which they have denotations as both arguments and predicates (Rothstein 2012, 2013, 2017; Scontras 2017). This duality parallels that assumed for nominal expressions, particularly bare plurals and mass terms, which are widely considered to have interpretations as both individuals (kinds) and properties. In both cases, the argument interpretation can be derived as the nominalization of the predicate; conversely, the predicate characterizes individuals that instantiate the nominalized property, whether it be an ordinary kind or a numerical degree. Scontras in particular shows that treating degrees as a sort of kind allows for an elegant semantic account of words like amount, which like kind-denoting expressions allow both direct and existential interpretations. He further shows that his analysis provides an account of some seemingly unrelated phenomena, such as so-called amount relatives (Carlson 1977).

Our analysis of the some+numeral construction provides further support for this view of degrees. Expressions such as twenty, five ounces, 45 minutes, 50 meters and 10 feet (longer) do not just denote points on a measurement scale. They can also be construed as sets of physical or abstract individuals: pluralities, portions of matter, temporal intervals, spatial extents and scale segments. It is this that allows them to be selected by some, whose first argument is always a set-denoting expression of some sort. Thus an investigation of this small corner of numerical semantics yields insights into the nature of degrees more generally.

At the same time, we also have evidence that not all numerical expressions have this more concrete set-based interpretation. We have argued that expressions of temporal location such as three o'clock and 1990 do not, as they do not compose with some, and also cannot serve as the restrictor for the quantifier every. Expressions of spatial location (e.g. 12,000 feet in (9c)) appear to belong to the same category. We suspect there are other cases as well. An example involves temperature expressions. According to our judgments, ?Yesterday was some 20 degrees Celsius is slightly ill-formed, suggesting that 20 degrees on this use cannot be interpreted as set denoting. While we do not have a definitive answer as to why this should be, we hypothesize that the reason might be that temperature measures of this sort - unlike those measures that can compose with some - are not monotonic on the part/whole structure of their domain of predication. Pursuing this hypothesis further must be left to future work. 
Finally, we saw earlier that in contexts such as mathematical statements, some is ruled out via a type clash: The context requires the argument interpretation of the numerical expression, but some can only compose with its predicative interpretation. If, as we assume here, the some of some+numeral is based on the same lexical entry as the indefinite determiner some, and if nominal expressions exhibit the same argument/predicate duality that degrees do, then we might expect there to be contexts in which nominal expressions likewise do not allow modification by some. In fact, we hypothesize this to be the explanation for the observation (Krifka 2004 and others) that some is disallowed in generic sentences, including both direct kind reference as in (44) and characterizing generics as in (45):

(\#Some) dinosaurs are extinct.

(on kind reference reading)

(\#Some) dogs bark.

(on generic reading)

An example such as (44) with a bare plural can be analyzed as involving the kind predicate extinct applying directly to the kind DINOSAUR. It is thus fully expected that composition with some is impossible. A similar explanation can be applied to cases like (45) if we assume that these too start with the kind-based interpretation of the plural nominal (Chierchia 1998; Cohen 2007). Thus the same property of some that precludes its occurrence with numerical values in mathematical statements can also account for restrictions on the occurrence of some in the nominal domain. This provides further support for treating indefinite determiners such as some as operators on quantificational domains, and for establishing a parallel between degrees and kinds.

\section{Conclusions}

The starting point of this paper was the observation that English some has a curious use on which it combines with numerical expressions. We have demonstrated that by adopting established proposals for the semantics of degree expressions and of indefinite determiners, it is possible to give a compositional analysis of the some+numeral construction that accounts for both the constraints on its distribution as well as the variable presence of an approximating effect. In this, our analysis has an advantage over previous treatments of the construction.

Looking more broadly, we have also argued that the facts from this domain support a view according to which numerical expressions have interpretations not just as abstract degrees on scales, but also as sets of (more or less concrete) individuals. And we have drawn a connection between numerical approximation and the domain shifting function that has been proposed to underlie the semantics of indefinite determiners more generally. We believe that these connections are important areas for further exploration. 
Some three students

\section{References}

Alonso-Ovalle, Luis \& Paula Menéndez-Benito. 2003. Some epistemic indefinites. Thirty-Third Annual Meeting of the North East Lingusitic Society (NELS 33) $1-12$.

Alonso-Ovalle, Luis \& Paula Menéndez-Benito. 2010. Modal indefinites. Natural Language Semantics 18. 1-31. doi:10.1007/s11050-009-9048-4.

Alonso-Ovalle, Luis \& Paula Menéndez-Benito. 2011. Plural epistemic indefinites. Fortieth Annual Meeting of the North East Linguistic Society (NELS 40) 17-30.

Alonso-Ovalle, Luis \& Paula Menéndez-Benito. 2013. Two views on epistemic indefinites. Language and Linguistics Compass 7(2). 105-112. doi:10.1111/lnc3.12009.

Altshuler, Daniel. 2014. Discourse transparency and the meaning of temporal locating adverbs. Natural Language Semantics 22. 55-88. doi:10.1007/s11050013-9100-2.

Anderson, Curt. 2014. Approximation of complex cardinals using some. Fortieth Western Conference On Linguistics (WECOL 2013) 131-143.

Anderson, Curt \& Marcin Morzycki. 2015. Degrees as kinds. Natural Language and Linguistic Theory 33(3). 791-828. doi:10.1007/s11049-015-9290-z.

Barwise, Jon \& Robin Cooper. 1981. Generalized quantifiers and natural language. Linguistics and Philosophy 4(2). 159-219. doi:10.1007/BF00350139.

Carlson, Greg N. 1977. Amount relatives. Language 53(3). 520-542. doi: $10.2307 / 413175$.

Chierchia, Gennaro. 1998. Reference to kinds across language. Natural Language Semantics 6(4). 339-405. doi:10.1023/A:1008324218506.

Chierchia, Gennaro. 2013. Logic in Grammar: Polarity, Free Choice and Intervention. Oxford: Oxford University Press. doi:10.1093/acprof:oso/9780199697977.001.0001.

Cohen, Ariel. 2007. Between kinds and properties: bare plurals across languages. Seventeenth Semantics and Linguistic Theory Conference (SALT17) 53-70.

Davies, Mark. 2008-. The Corpus of Contemporary American English (COCA): 560 million words, 1990-present. Available online at http://corpus.byu.edu/coca/.

Faller, Martina. 2000. Dimensional adjectives and measure phrases in Vector Space Semantics. In Martina Faller, Stefan Kaufmann \& Marc Pauly (eds.), Formalizing the Dynamics of Information, 151-170. Stanford: CSLI Publications.

Geurts, Bart. 2006. Take 'five': the meaning and use of a number word. In Svetlana Vogeleer \& Liliane Tasmowski (eds.), Non-Definiteness and Plurality, 311-329. Amsterdam and Philadelphia: John Benjamins. doi:10.1075/la.95.16geu.

Heim, Irene. 1982. The semantics of definite and indefinite noun phrases. Amherst, MA: University of Massachusetts PhD dissertation. 
Horn, Laurence R. 1984. Toward a new taxonomy for pragmatic inference: Q-based and R-based implicature. In Deborah Shiffrin (ed.), Meaning, Form and Use in Context: Linguistic Applications, 11-89. Washington, D.C.: Georgetown University Press.

Ionin, Tania \& Ora Matushansky. 2006. The composition of complex cardinals. Journal of Semantics 23(4). 315-360. doi:10.1093/jos/ffl006.

Kadmon, Nirit \& Fred Landman. 1993. Any. Linguistics and Philosophy 16(4). 353-422. doi:10.1007/bf00985272.

Kennedy, Christopher. 2015. A “de-Fregean” semantics (and neo-Gricean pragmatics) for modified and unmodified numerals. Semantics and Pragmatics 8(10). 1-44. doi:10.3765/sp.8.10.

Kratzer, Angelika. 2005. Indefinites and the operators they depend on: From Japanese to Salish. In Gregory N. Carlson \& Francis Jeffry Pelletier (eds.), Reference and Quantification: The Partee Effect, vol. 173 CSLI Lecture Notes, 113-142. Stanford: Center for the Study of Language and Information.

Kratzer, Angelika \& Junko Shimoyama. 2002. Indeterminate pronouns: the view from Japanese. Third Tokyo Conference on Psycholinguistics 1-25.

Krifka, Manfred. 1989. Nominal reference, temporal constitution and quantification in event semantics. In Renate Bartsch, Johan van Benthem \& Peter van Emde Boas (eds.), Semantics and contextual expressions, 75-115. Dordrecht: Foris.

Krifka, Manfred. 2002. Be brief and be vague: and how bidirectional optimality theory allows for verbosity and precision. In David Restle \& Dietmar Zaefferer (eds.), Sounds and Systems. Studies in Structure and Change: A Festschrift for Theo Vennemann, 439-458. Berlin: Mouton de Gruyter.

Krifka, Manfred. 2004. Bare NPs: Kind referring, indefinites, both, or neither. Empirical Issues in Syntax and Semantics 5. 111-132.

Krifka, Manfred. 2007. Approximate interpretations of number words: A case for strategic communication. In Gerlof Bouma, Irena Krämer \& Joost Zwarts (eds.), Cognitive Foundations of Interpretation, 111-126. Koninklijke Nederlandse Akademie van Wetenschappen.

Landman, Fred. 2004. Indefinites and the Type of Sets. London: Blackwell.

Lasersohn, Peter. 1999. Pragmatic halos. Language 75(3). 522-551. doi: $10.2307 / 417059$.

Nouwen, Rick. 2010. Two kinds of modified numerals. Semantics and Pragmatics 3(3). 1-41. doi:10.3765/sp.3.3.

Rothstein, Susan. 2011. Counting, measuring and the semantics of classifiers. The Baltic International Yearbook of Cognition, Logic and Communication. 6.

Rothstein, Susan. 2012. Numericals: Counting, measuring and classifying. Sinn und Bedeutung 16 527-543. 
Some three students

Rothstein, Susan. 2013. A Fregean semantics for number words. Nineteenth Amsterdam Colloquium 179-186.

Rothstein, Susan. 2017. Semantics for Counting and Measuring Key Topics in Semantics and Pragmatics. Cambridge: Cambridge University Press.

Sauerland, Uli \& Penka Stateva. 2007. Scalar vs. epistemic vagueness: evidence from approximators. Seventeenth Semantics and Linguistic Theory Conference (SALT17) 228-245.

Schwarzschild, Roger. 2002. Singleton indefinites. Journal of Semantics 19(3). 289-314. doi:10.1093/jos/19.3.289.

Scontras, Gregory. 2017. A new kind of degree. Linguistics and Philosophy 40(2). 165-205. doi:10.1007/s10988-016-9200-9.

Solt, Stephanie. 2014. An alternative theory of imprecision. TwentyFourth Semantics and Linguistic Theory Conference (SALT24) 514-533. doi:10.3765/salt.v24i0.2446.

Stevens, Jon \& Stephanie Solt. 2018. The semantics and pragmatics of "some 27 arrests". University of Pennsylvania Working Papers in Linguistics 24(1). $179-188$.

Weir, Andrew. 2012. "Some”, speaker knowledge, and subkinds. European Summer School in Logic, Language and Information (ESSLLI) 2012 Student Session 180-190.

Winter, Yoad. 2001. Measure phrase modification in a vector space semantics. Twentieth West Coast Conference on Formal Linguistics (WCCFL 20) 607-620.

Zwarts, Joost. 1997. Vectors as relative positions: A compositional semantics of modified PPs. Journal of Semantics 14(1). 57-86. doi:10.1093/jos/14.1.57.

Zwarts, Joost \& Yoad Winter. 2000. Vector space semantics: a model-theoretic analysis of locative prepositions. Journal of Logic, Language and Information 9. 169-211.

Stephanie Solt

Leibniz-Zentrum Allgemeine Sprachwissenschaft (ZAS)

Schützenstraße18

10117 Berlin, Germany

solt@leibniz-zas.de
Jon Stevens

Department of Linguistics

The Ohio State University Columbus, OH 43085, USA

jonscottstevens@gmail.com 